\title{
On calcium fluorophore's impact in platelet signaling studies
}

\author{
by Sofia V. Galkina1, and Fedor A. Balabin ${ }^{2,3 \#}$
}

1. Faculty of Physics, Lomonosov Moscow State University, 1/2 Leninskie gory, Moscow, 119991, Russia

2. Center for Theoretical Problems of Physico-Chemical Pharmacology, Russian Academy of Sciences, 30 Srednyaya Kalitnikovskaya str., Moscow, 109029, Russia

3. National Medical Research Center of Pediatric Hematology, Oncology and Immunology named after Dmitry Rogachev, 1 Samory Mashela St, Moscow, 117198, Russia

\# Correspondence: fa.balabin@physics.msu.ru

Received: 15.12.2021

Accepted: 29.12.2021

Published: $\mathbf{3 0 . 1 2 . 2 0 2 1}$

DOI: 10.52455/sbpr.01.202104012

\section{Abstract}

Observation of calcium signaling in platelets - blood cells designed to be involved in stopping bleeding and forming blood clots - is an important part of fundamental research in hemostasis. However, such a study is possible only with the use of calcium fluorophores - small molecules that penetrate the platelet membrane due to their hydrophobic -AM part, which is then hydrolyzed by cytosol esterases. In this work, we consider the phenomenon of inhomogeneous loading of calcium fluorophores into platelets.

We used platelets from healthy adult donors loaded with various fluorescent probes (CalBryte590, DiOC6 (3), Fura Red, Fluo-4 and CellTracker Violet BMQC) and immobilized on antibodies to CD31 in parallel plane flow chambers. Total internal reflection fluorescence (TIRF) microscopy was used for observations.

We demonstrated that all studied probes are loaded heterogeneously, with 30\% platelets being loaded with a probe 2-6 times higher than the population median value. Using the CalBryte590 probe as an example, we have shown that a decrease in the incubation temperature, the addition of Pluronic 127 to the incubation medium, or membrane cholesterol depletion significantly reduces the heterogeneity of the probe distribution in the population. By looking at platelet activation from the surface, we have shown that the probability of experiencing strong activation, as measured by the intensity of calcium oscillations, correlates with the amount of probe in the platelet.

Thus, we conclude that the type of fluorophore used and the conditions of its loading into platelets can significantly affect the results of experiments on the observation of calcium signaling in platelets.

Keywords: intracellular signaling; fluorescent phrobes; membrane; calcium fluorophores; platelets

\section{Introduction}

Blood is an important component of the human body, however, a system for maintaining its integrity is needed - a hemostasis system, roughly divided into plasma and vascular-platelet links [1]. Platelets, small non-nuclear blood cells, are designed to stop bleeding when a vessel is damaged by forming an aggregate and providing its surface to accelerate the work of the plasma hemostasis link [2]. To accomplish this task, platelets enter the so-called activated state. When activated, platelets adhere to the surface and aggregate with each other [3], change their shape, secrete the contents of granules [4] and, in some cases, become procoagulant $[5,6]$. Eventually, a blood clot forms. [7].

A feature of platelet activation is its quick reaction to damage - with characteristic times of the order of one second [8]. Probably, the platelet intracellular signaling system provides such a response rate due to calcium ions. In resting cells, the concentration of free calcium in the cytosol is maintained at a lower level (approximately 10-20 nM) [9] than in blood plasma (1-2 mM), and in the endoplasmic reticulum, which is considered the main store of calcium in the platelet, its concentration is about $100-800 \mu \mathrm{M}$ [10]. Platelet activation, like many other cells, is controlled by the concentration of calcium ions in the cytosol due to the fact that calcium ions are cofactors for many enzymes mediating platelet activation [11-13].

As such an important component of platelet activation, calcium signaling has been studied for a long time both in cell suspension $[14,15]$ and in single platelets [16-18]. The study of calcium signaling is carried out using intracellular fluorescent probes - small organic molecules-fluorophores, the parameters of excitation and / or fluorescence of which significantly change when binding calcium ions [19]. However, without damaging the cell membrane, fluorescent probes can be introduced into it only by diffusion, and for this the molecules are modified by the addition of an acetomethyl residue, which is hydrolyzed inside the cell by nonspecific esterases [20]. 
In this work, using single human platelets, we investigated the issues of the possible influence of the used fluorescent probe and the conditions of its entry into the cell on the observed calcium signal. As a result of this work, we conclude that the intensity of calcium mobilization in the platelet in response to activation correlates positively with the level of fluorescence of the fluorophore in the platelet.

\section{Materials and methods}

The following materials were obtained from the parenthesized sources: Human Thrombin (Hematologic Technologies, Essex Junction, Vermont, USA), Calbryte 590 AM, Fura Red AM, Fluo$4 \mathrm{AM}$, and DiOC6 (3) (Molecular probes, Eugene, State Oregon, USA), CellTracker Violet BMQC Dye (Invitrogen), S-Monovette Hirudin $1.6 \mathrm{ml}$ hirudin tubes (SARSTEDT AG Co. KG, Germany), $4.5 \mathrm{ml}$ citrate (3.8\%) tubes (IMPROVACUTER, China). Potato apyrase, ADP and solution reagents were obtained from Sigma-Aldrich. VM64 antibodies were given as a gift to Prof. A.V. Mazurov (Federal State Budgetary Institution of National Medical Research Center of Cardiology, Moscow, RF). Materials for the manufacture of flow chambers were produced by GemaKor (Moscow, RF) and MedSil (Mytishchi, RF).
The whole blood of healthy volunteers (11 persons, 18-45 years of age) within 3 hours of the collection was used for all experiments. Blood was collected into $9 \mathrm{ml}$ tubes containing 3,8\% sodium citrate $(1: 9 \mathrm{v} / \mathrm{v})$, or into $1.6 \mathrm{ml}$ tubes containing R-hirudin (S-Monovette ${ }^{\circledR}$, Sarstedt, Germany). Investigations were performed in accordance with the Declaration of Helsinki and approved by the CTP PCP RAS ethics committee (decision \#1 from 12.01.2018), written informed consent was obtained from all donors.

9 different protocols (A-I) for loading calcium sensitive probes into platelets were used for this study. Protocols A-E are based on the sodium citrateanticoagulated $(3.8 \% \mathrm{v} / \mathrm{v})$ blood, while in protocols F-H hirudin (> 525 antithrombin units $/ \mathrm{ml}$ ) was used as the anticoagulant. $2 \mu \mathrm{M}$ of Calbryte590-AM was used as a probe for protocols $\mathrm{A}, \mathrm{B}, \mathrm{D}, \mathrm{E}$, and $\mathrm{H}$, protocol $\mathrm{C}$ used DIOC6, protocol F - Fluo4-AM $(2 \mu \mathrm{M})$, protocol G - Fura Red-AM $(2 \mu \mathrm{M})$, and protocol I - CellTracker Violet BMQC Dye $(2 \mu \mathrm{M}$ and $4 \mu \mathrm{M})$. Apyrase $(0.3 \mathrm{U} /$ $\mathrm{ml}$ ) was used to prevent platelet pre-activation due to ADP, secreted by the red blood cells. Protocol $D$ also contained Pluronic F-127 (0.04\%) and in protocol E, $5 \mathrm{mM}$ of $\mathrm{M} \beta \mathrm{CD}$ (methyl- $\beta$-cyclodextrin) was added after 15 min of blood incubation with calcium probe.

\section{DIC DIC DIC, TIRF $561 \mathrm{HM}$}

$\begin{array}{lll}\text { Whole blood } & \text { Unbound cells } & \text { Filming the } \\ \text { enters flow } & \text { are washed } & \text { experiment } \\ \text { chamber } & \text { with buffer } & \text { on video }\end{array}$

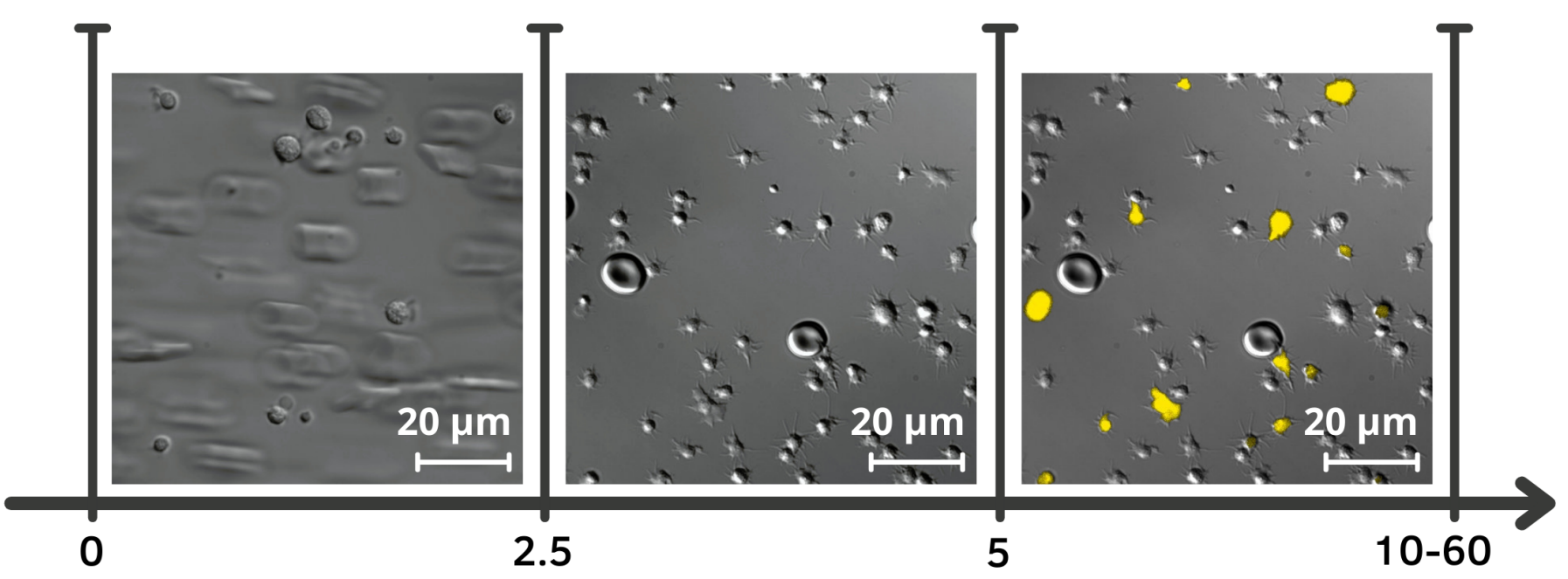

Experiment duration, $\min$

Figure 1. Overall scheme of the assay. First, whole pre-processed blood is perfused through the flow chamber for 2.5 minutes. Then Tyrode's buffer is perfused through the chamber for 5 minutes to wash away unattached cells. Then, depending on the protocol, the microscopy video is recorded for 5-10 minutes. 
The resulting mixture was incubated for 30 minutes $(A, B, D, E, F, G, H)$ or for 5 minutes $(C)$ at $37^{\circ} \mathrm{C}(A, D$, $E, F, G, H)$ or at $25^{\circ} \mathrm{C}(B, C)$. Thus, protocol $A$ can be used as a control for the CalBryte590 dye, protocol $\mathrm{C}$ for the DiOC6 dye, $\mathrm{F}$ for the Fluo4 dye, $\mathrm{G}$ for the Fura Red dye, and I - CellTracker Violet BMQC dye. Protocols A, B, D and E use different conditions for loading the CalBryte590 probe into platelets: B is characterized by a reduced temperature, $D$ by the presence of Pluronic (a reagent to facilitate the entry of a hydrophobic AM probe into the cell), and Protocol $E$ is characterized by the presence of $M \beta C D$ (reagent for depletion platelet membrane with cholesterol).

Parallel-plate flow chambers, used here, were described earlier [21]. Monoclonal antibody to CD31 covered glass coverslips were used for platelet immobilization. Anti-CD31 antibody was isolated same as in [22]. Platelets were isolated from the whole blood upon perfusion through the flow chamber and interaction with the antibody covered glass. Unattached cells were washed away using tyrode's calcium buffer and then platelet activation was monitored upon perfusion of the buffers, containing platelet activators. Nikon Eclipse Ti-E microscope with a CFI Apochromat TIRF 100XC Oil objective with 100-fold magnification and a numerical aperture of 1.49 was used in this study. Fluorescent probes were excited by laser at 405, 488 and $561 \mathrm{~nm}$ wavelength. Images were recorded using an Andor iXon3 EMCCD camera. The scheme of the experiment is given in Figure 1.

ImageJ-Fiji was used for the analysis of the experimental data. Regions of interest (ROIs) of $2 \mu \mathrm{m}$ diameter were selected inside of the cells. At each timestep averaged fluorescence intensity was measured inside these ROls. Based on this data, fluorescence intensity profiles were built (Fig. 2). Pre-activation of platelets has been assessed by identification of specific platelet types within the field of view (FOV) as has been previously described [23]: (1) weak activation (less than five random single peaks per minute), (2) activation (multiple stochastic peaks, which usually do not form clusters ), (3) strong activation (clusters of peaks in the profile) and (4) a stably high response (changes in fluorescence intensity are mainly caused by bleaching or leakage of the fluorescent probe) (Fig. 2).
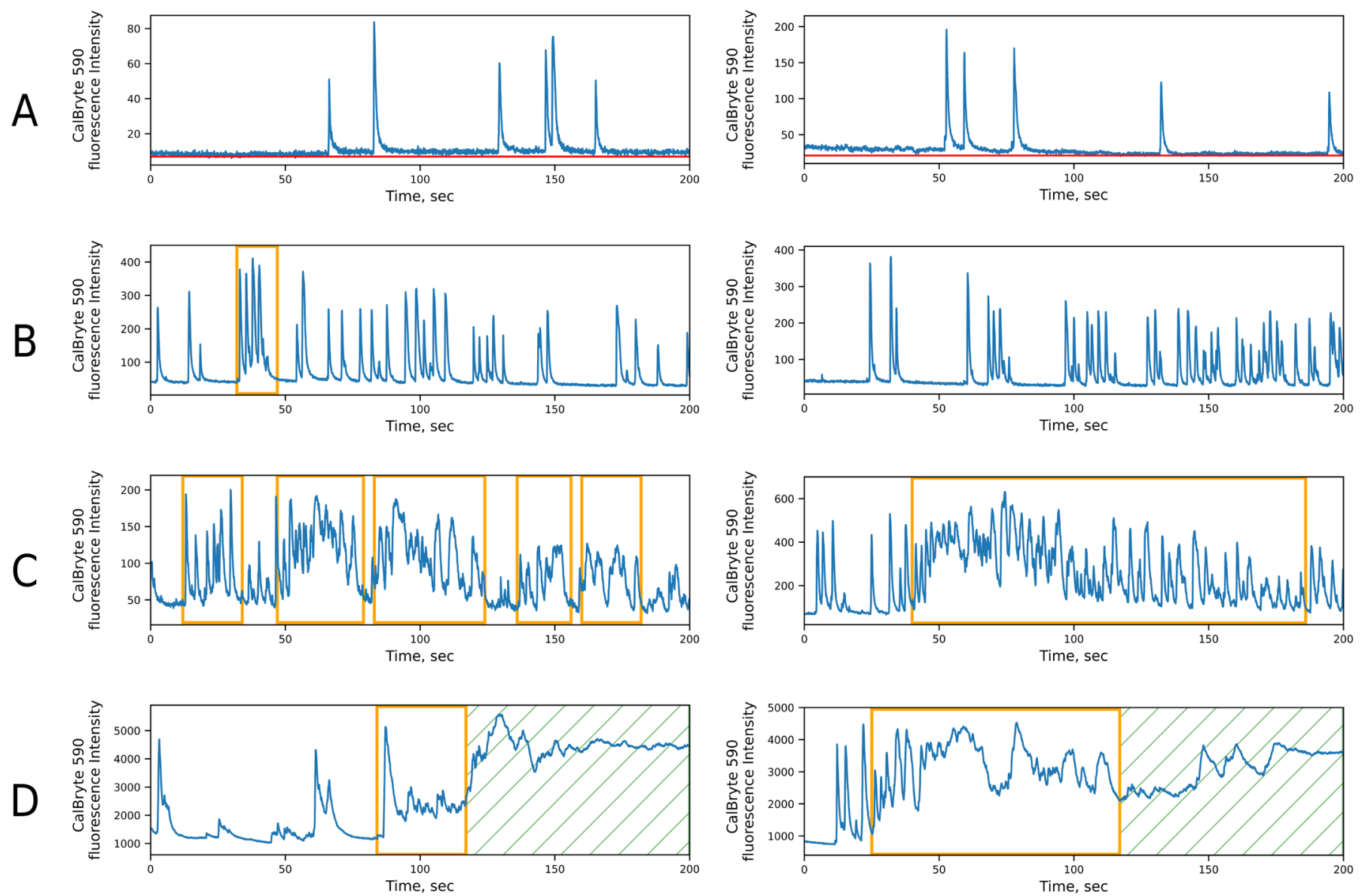

Figure 2. Observed fluorescence intensity profiles of individual platelets and their classification. (A) Group 1: the cell shows less than five random single peaks in 60 seconds of observation. The red line shows the baseline fluorescence intensity level; (B) Group 2: platelets are weakly activated, there are multiple stochastic peaks, which usually do not form clusters; (C) Group 3: platelet activation is intense, peaks are so frequent that individual peaks coalesce into clusters; (D) Group 4: Presumably, the cells show a persistently high concentration of calcium, any observed changes in fluorescence intensity are mainly caused by bleaching or leakage of the fluorescent dye; Orange boxes are clusters of peaks (clusters). Green shaded areas are persistent high calcium levels. 


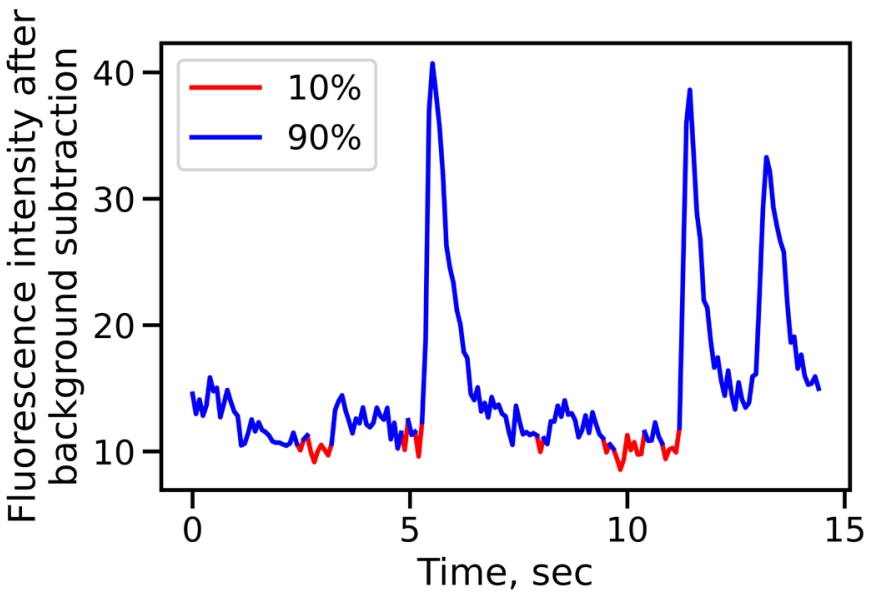

Figure 3. Example of selecting the $10 \%$ of the points with the lowest intensity in a fluorescence intensity profile.

The amount of fluorophore, "loaded" into the platelet, was calculated as the average fluorescence of $10 \%$ of the points with the lowest fluorescence intensity (Fig. 3).

\section{Results and Discussion}

Platelets are loaded heterogeneously by different fluorescent dyes

Different dyes load to platelets heterogeneously. The amount of dye in platelets of the same donor varies between the cells. To study this phenomenon, we determined the specificity of each probe. Platelets were immobilized on VM64 (anti-CD31). The amount of the probe in the platelet was assessed by the profile in the first 15 seconds of the experiment. Platelets from the same donor were loaded with four different fluorescent probes: CalBryte 590-AM (Protocol H), DiOC6(3) (Protocol C), Fura Red-AM (Protocol F) and Fluo-4-AM (Protocol G) (Fig. 4). For all dyes, there was a heterogeneity of the base platelet fluorescence
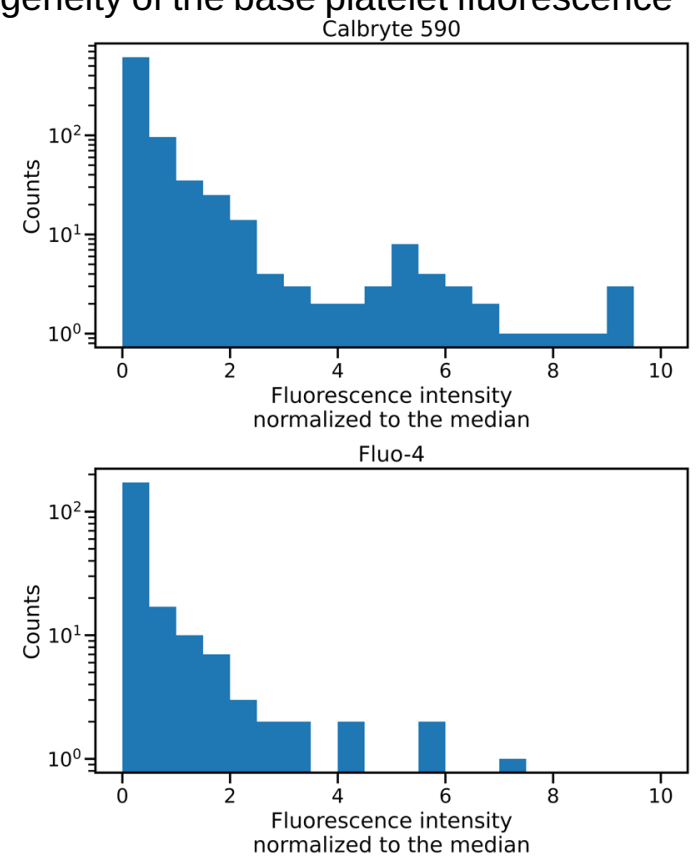

intensity with a deviation of the fluorescence intensity from the median value by $2-6$ times for $30 \%$ of the population. Interestingly, Calbryte 590-AM (Fig. 4A) and Fluo-4-AM (Fig. 4C) load more homogeneously than DiOC6 (Fig. 4B) and Fura Red-AM (Fig. 4D).

The observed phenomenon of specific loading of different probes may be related to the size and polarity of the fluorescent probe molecules. The presence of a difference in a potential of the platelets membrane $[24,25]$ facilitates the entry of cationic probes and hinders the anionic ones. However, heterogeneity of potential in a population can lead to heterogeneity of loading. Since DiOC6 (3) is a voltage-sensitive probe that does not contain the -AM part, its distribution in the platelet population precisely reflects this property of the platelet. (Fig. 4B). It has already been shown that Fluo-4 distributes heterogeneously in the platelets [26] and it has been primarily associated with the compartmentalization of the dye. However, the causes for this heterogeneity are not fully understood. Perhaps, the compartmentalization of the dye may cause heterogeneity. Furthermore, it is known that low temperature of incubation and the usage of surfactants reduce the compartmentalization of the probes [27].

In order to find out the cause for the uneven loading of platelets with fluorescent probes, we used the CellTracker Violet BMQC dye (Figure 5). Our purpose was to visualize the position of the cell in space. This substance is a derivative of coumarin, it can freely enter the cell and remain inside it for a long time because of binding with glutathione. We have no data that the fluorescence intensity of this dye depends on the concentration of calcium in the cytosol, hence it follows that the state of the plasma membrane plays the main role in the ingress of the dye into the cell.
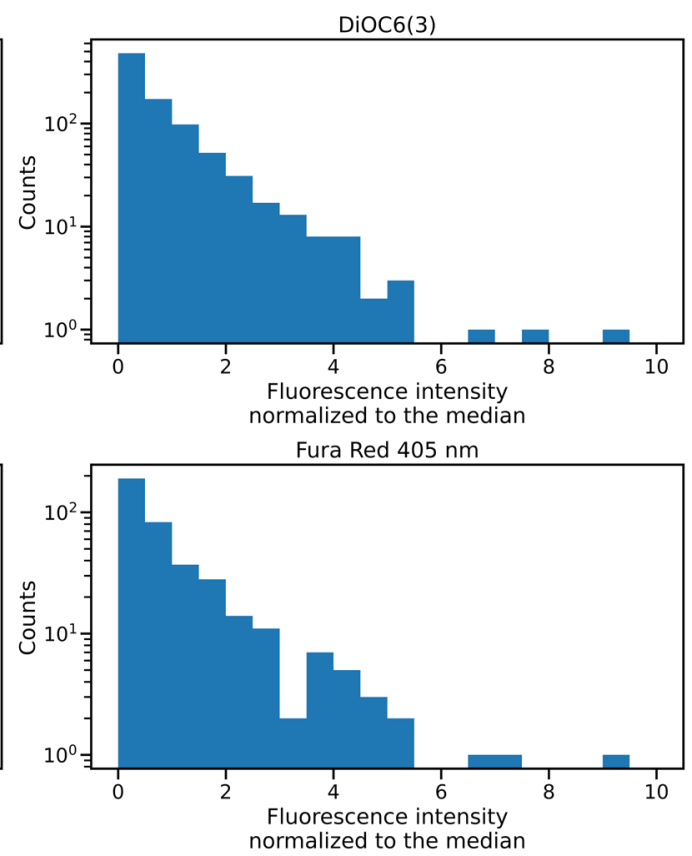

Figure 4. Baseline fluorescence intensity histograms for CalBryte 590 AM, DiOC6 (3), Fluo-4 AM and Fura Red. The fluorescence of the probes is normalized to the median. Each histogram is based on data analysis of $n=270$ platelets from $\mathrm{m}=3$ healthy donors 


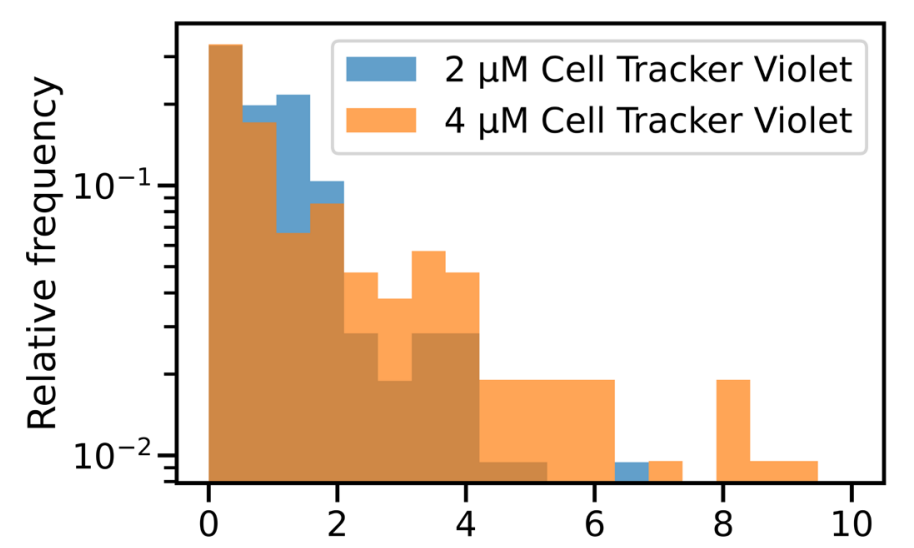

Fluorescence intensity normalized to the median

Figure 5. Histograms of baseline fluorescence intensity for the CellTracker Violet BMQC probe for 2 $\mu \mathrm{M}$ and $4 \mu \mathrm{M}$, normalized to median.

Platelet loading with a fluorescent probe depends on the experimental conditions

The loading of the fluorescent dye may be affected by the conditions of incubation. To test this, we compared the amount of probe in the platelet under different loading conditions: at room temperature (protocol B, see Methods), in the presence of a hydrophobic agent (protocol D) or when cholesterol is depleted from the membrane (protocol E) (Fig. 6). The statistical significance of differences in the number of "loaded" probe was calculated using the Mann-Whitney and Kolmogorov-Smirnov tests (Table 1).

All three samples do not belong to the same distribution law as the control one. Samples M $B C D$ and $25^{\circ} \mathrm{C}$ for both tests give a p-value of less than 0.01, a sample with Pluronic F-127 for the

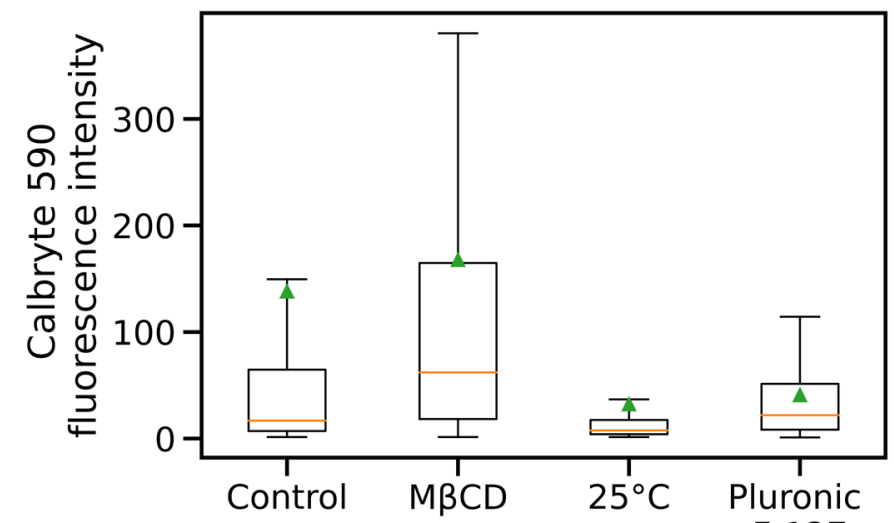

F-127

Figure 6. Dependence of the level of basic fluorescence intensity on the conditions of the experiment. Green arrow - mean value, orange bar - median value, "box" - from $25 \%$ to $75 \%$ of the population, "wiskers" - $95 \%$ confidence interval. $M B C D$ increases the fluorescence intensity of the Calbryte 590 AM probe compared to the control. A decrease in the activation temperature from $37^{\circ} \mathrm{C}$ to $25^{\circ}$ C slows down the loading of cells with the probe. Pluronic F-127 forces the probe to spread more evenly throughout the cells than under control conditions, but does not affect the platelet membrane itself. Data are given for $n=360$ platelets from $m=10$ healthy donors.
Kolmogorov-Smirnov test gives a $p$-value of less than 0.01 , and for the Mann-Whitney test gives a $p$-value of less than 0.05 .

Table 1. Comparison of the parameters of the control group with the rest.

\begin{tabular}{|l|c|l|l|l|}
\hline & Control & MBCD & $25^{\circ} \mathrm{C}$ & Pluronic F127 \\
\hline Mean & 138.1 & $167.7(121.4 \%)$ & $32.2(23.3 \%)$ & $40.7(29.5 \%)$ \\
\hline Median & 16.8 & $62.1(369.6 \%)$ & $7.6(45.2 \%)$ & $21.9(130.4 \%)$ \\
\hline $2.5 \%$ percentile & 2.3 & $3.4(147.8 \%)$ & $2.0(87.0 \%)$ & $2.8(121.7 \%)$ \\
\hline $25 \%$ percentile & 7.1 & $18.3(257.7 \%)$ & $4.0(56.3 \%)$ & $8.2(115.5 \%)$ \\
\hline $75 \%$ percentile & 64.6 & $164.8(255.1 \%)$ & $17.4(26.9 \%)$ & $51.3(79.4 \%)$ \\
\hline $97.5 \%$ percentile & 1084.0 & $909.5(83.9 \%)$ & $225.8(20.8 \%)$ & $162.1(14.9 \%)$ \\
\hline
\end{tabular}

Incubation at room temperature, compared with incubation at $37^{\circ} \mathrm{C}$, reduces the average and median fluorescence intensities of cells by more than $100 \%$. This result is consistent with the data that the permeability of the cell membrane is dependent on temperature [28].

Pluronic F-127 reduces the average amount of the probe in the platelet (by 240\%) and the median (by $23 \%$ ). The upper limit of the $95 \%$ confidence interval is decreased by more than $550 \%$. Pluronic is a surfactant that increases the solubility of probes in the AM form [29]. Thus, the distribution of the probe in the cell becomes more uniform, and the number of overloaded platelets decreases (mean value decreases, the median value does not change).

Incubation with $M \beta C D$ increases the mean (by $17.7 \%$ ) and median (by $72.9 \%$ ) values of the fluorescence intensity in comparison with the control sample. $M \beta C D$ increases platelet membrane fluidity by decreasing cholesterol concentration [30], while not leading to cell activation [31,32]. The observed values of the amount of the probe show that M $\beta C D$ promotes the dye to pass into the cell. That leads to a suggestion that the amount of the probe depends on the state of the membrane.

\section{$M \beta C D$ shortens the time of loading cells with a fluorescent probe}

Cholesterol of the membrane affects the loading of the platelets with a probe. To investigate this, the platelet loading rate was monitored with a Calbryte 590-AM fluorescent probe without M $B C D$ (Protocol A) and in presence of $M \beta C D$ (Protocol E) (Fig. 7). $M \beta C D$ increases the rate of entry of the probe into the cell by approximately 2 times. And the increase in fluorescence intensity in the cytosol begins 2 times faster than under control conditions.

The amount of the probe in the platelet correlates with its response to stimulation

The fluorescent probe does not evenly enter platelets in the population regardless of the dye and loading conditions. But it remains unclear whether this phenomenon is for the study of calcium signaling in platelets. To investigate this, we checked 


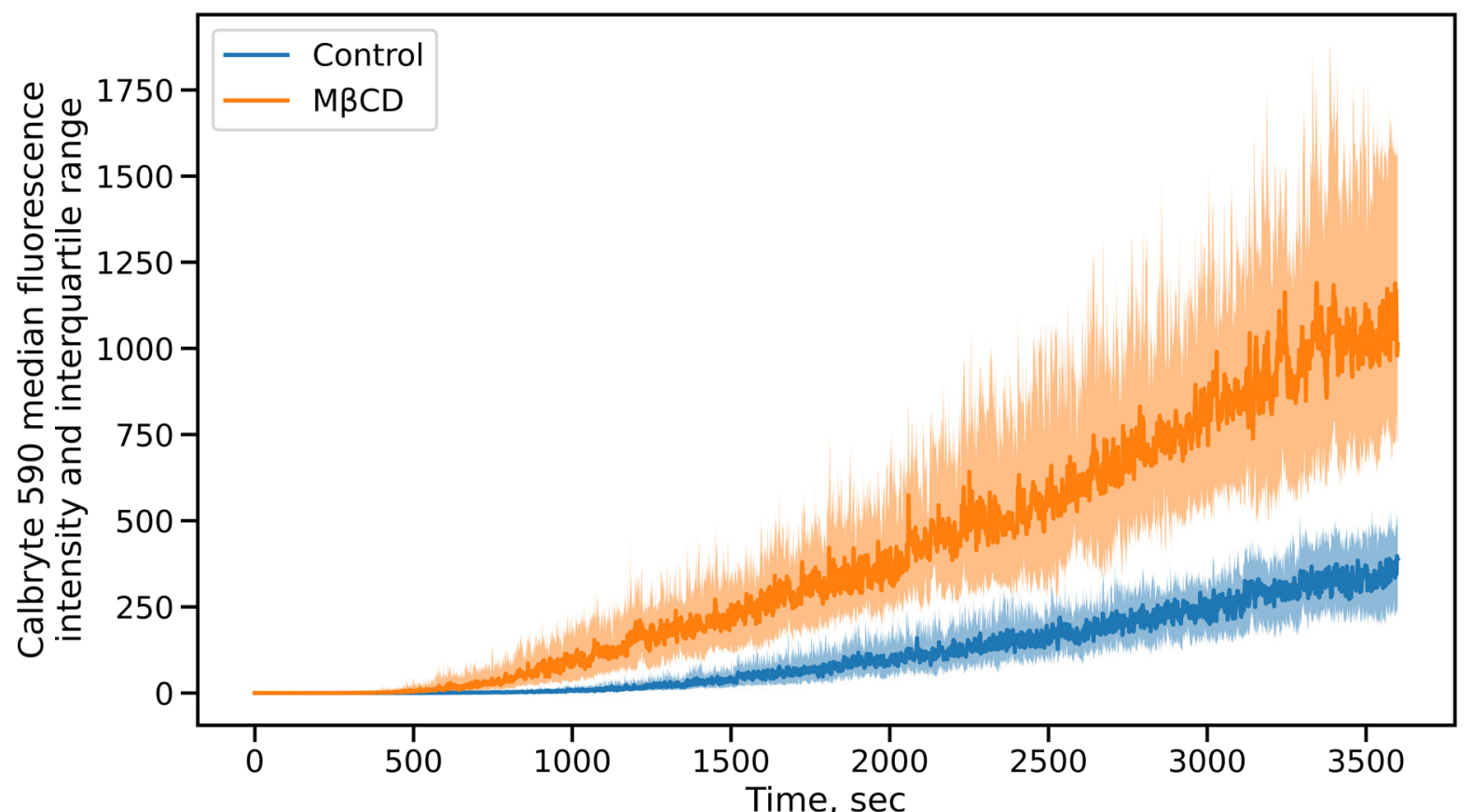

Figure 7. Comparison of median fluorescence intensity versus time in the presence and absence of $M \beta C D$. Orange - with $M \beta C D$, blue - without $M \beta C D$. Light orange and light blue are the interquartile ranges respectively. Data are presented for $n=90$ platelets from $m=3$ healthy donors.

if the baseline fluorescence intensity of the probe and activation of platelet are related (Fig. 8). The amount of the probe in the platelet was assessed according to the profile in the first 15 seconds of the experiment. The division of platelets into groups by activation was proceeded according to the profiles taken within 5 minutes. Figure 8 shows the obtained dependence of the activation group on the amount of probe (the "lower limit" of the fluorescence intensity of CalBryte590 in the cell). It turned out that platelets from the fourth activation group had, on average, 10 times more probe than platelets from the third group. This can be explained by the possible activation of platelets of the fourth group at the time of the beginning of observation. However, platelets from the third activation group also had 7 times more probe than platelets from the second group. The second group had 1.5 times more probe than platelets from the first group. Since platelets of groups 1-3 have a pronounced baseline level (Fig. 2), we conclude that the degree of platelet activation correlates with the amount of fluorophore.

To clarify the observed phenomenon, we decided to estimate the probability of obtaining a platelet with a given value of baseline fluorescence in each of the activation groups using the available sample (Fig. 9). A platelet with a high baseline fluorescence intensity is more likely to undergo "strong" activation than a platelet with a low baseline fluorescence intensity.

Thus, it can be argued that evaluation of the response of platelets by measuring calcium concentration (by means of flow cytometry or spectrofluorimetry) is shifted in suspension. This shift occurs because the platelets, that are strongly activated, come to the fore. Thus, the overall response of the suspension appears to be stronger than under physiological conditions.

\section{Conclusion}

We have shown that platelets load heterogeneously with CalBryte 590 AM. This heterogeneity is not accidental. It was found that the probability of experiencing "strong" activation for a platelet with a high baseline fluorescence intensity is higher than for a platelet with a low baseline fluorescence intensity. The heterogeneity of platelet loading with a probe, as well as the loading process itself, can be influenced by various agents and experimental conditions. We observed this with the CalBryte 590 AM fluorescent dye. Incubation of the cells with the probe on $25^{\circ} \mathrm{C}$ instead of on $37^{\circ} \mathrm{C}$ slows down the loading. Pluronic F-127 makes the probe distribute more homogeneously throughout the cells

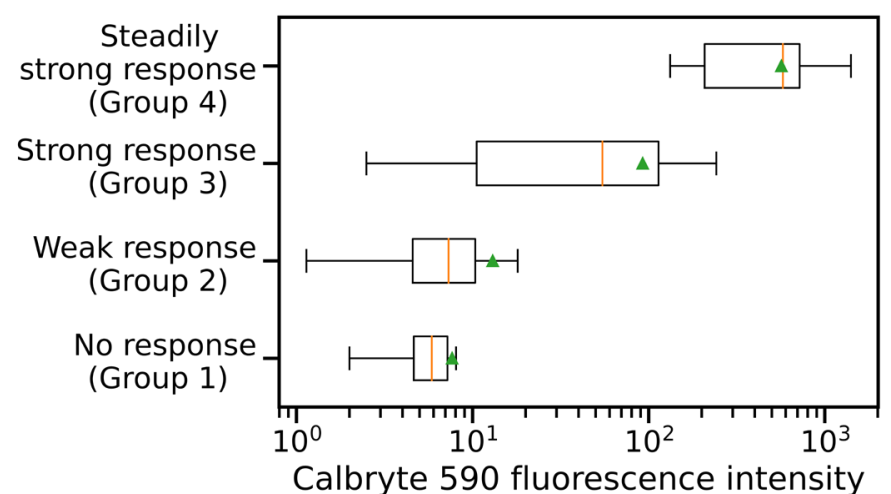

Figure 8. Dependence of the cell response group number on the intensity of its base fluorescence. Green arrow - mean value, orange line - median value, "box" - from $25 \%$ to $75 \%$ of the population, "whiskers" $95 \%$ confidence interval. In total, $\mathrm{n}=122$ platelets from $\mathrm{m}$ $=9$ healthy donors were analyzed. 


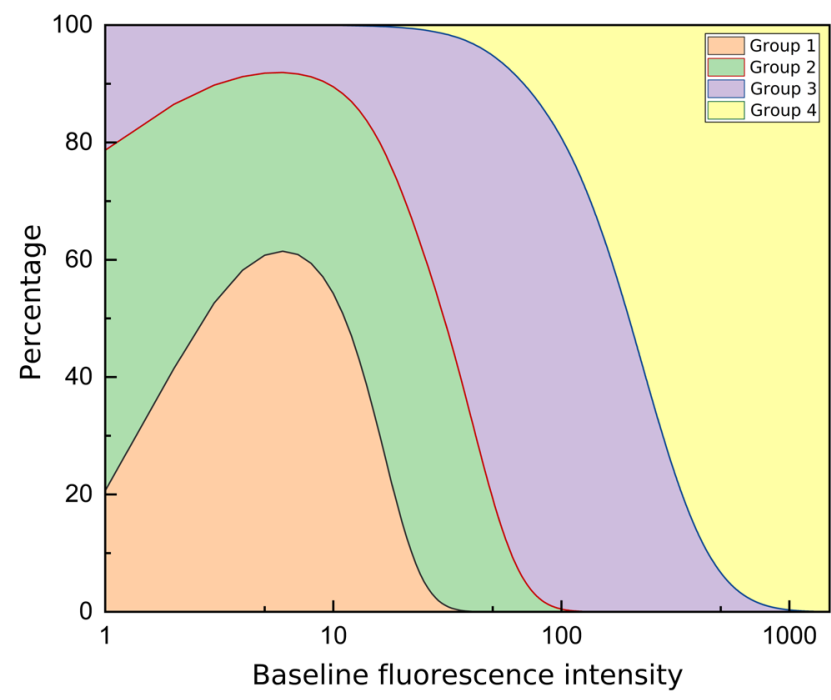

Figure 9. The probability of a platelet getting into the activation group, depending on the "lower limit" of the fluorescence intensity. The probability is calculated on the basis of data on $n=122$ platelets from $m=9$ healthy donors.

and it does not affect the platelet membrane. M $B C D$ increases the fluorescence intensity of the probe in the cytosol compared to control. It also increases the rate of platelet loading by the probe.

Heterogeneous cell loading is not unique to the CalBryte 590 AM probe. We have shown that fluorescent probes DiOC6 (3), Fura Red AM, Fluo$4 \mathrm{AM}$ also load heterogeneously into platelets. The heterogeneity of DiOC6 loading is comparable to that of other probes and it indicates that there is a noticeable heterogeneity of the potential difference on platelet membranes. It is assumed that this partially explains the phenomenon of heterogeneity of cell loading in general.

However, our data are insufficient to determine the cause for the heterogeneity of platelet loading to occur. A further investigation is required to study the combinations of the effects of the parameters that we used. Furthermore, size of the probe molecule, its quantum-chemical properties are of high interest to be investigated. However, it is possible to identify a number of factors that significantly affect the amount of a fluorescent probe in a living cell. First of all, the composition of the plasma membrane is important. We showed that hydrophobic low molecular weight substances pass through the membrane when it contains more unsaturated fatty acids. The amount of the loaded dye depends on the temperature of incubation. Secondly, the physicochemical state of the probe outside the cell has an effect. Moreover, the activity of nonspecific esterases, which cut off the AM"tails", is important. There are also two more factors that are sensitive to cell activation: the presence of nonspecific channels or carriers for both the AM-form of the probe and the working form, and the sensitivity of one of the probe forms to the potential difference on the membrane as a result of its charge.

\section{Aknowledgements}

The reported study was funded by RFBR and the Royal Society of London (RS), project number 2151-10005

\section{Author Contributions}

S.V.G. Performed fluorescent microscipy experiments, analyzed the data and wrote the paper; F.A.B. planned the study analyzed the data and edited the paper. All authors have read and agreed to the published version of the manuscript. The authors declare no conflict of interests.

\section{References}

1. Panteleev MA, Dashkevich NM, Ataullakhanov FI. Hemostasis and thrombosis beyond biochemistry: roles of geometry, flow and diffusion. Thrombosis Research 2015;136:699-711. https://doi.org/10.1016/j. thromres.2015.07.025.

2. Panteleev MA, Sveshnikova AN. Platelets and hemostasis. Oncohematology 2014;9:65-73.

3. Kaneva VN, Martyanov AA, Morozova DS, Panteleev MA, Sveshnikova AN. Platelet Integrin allbß3: Mechanisms of Activation and Clustering; Involvement into the Formation of the Thrombus Heterogeneous Structure. Biochem Moscow Suppl Ser A 2019;13:97110. https://doi.org/10.1134/S1990747819010033.

4. Sveshnikova $A$, Stepanyan $M$, Panteleev $M$, Sveshnikova A, Stepanyan M, Panteleev M. Platelet functional responses and signalling: the molecular relationship. Part 1: responses. Systems Biology and Physiology Reports 2021;1:20. https://doi.org/10.52455/ sbpr.01.202101014.

5. Topalov NN, Kotova YN, Vasil'ev SA, Panteleev MA. Identification of signal transduction pathways involved in the formation of platelet subpopulations upon activation. BrJHaematol 2012;157:105-15.

6. Podoplelova NA, Sveshnikova AN, Kotova YN, Eckly A, Receveur N, Nechipurenko DYu, et al. Coagulation factors bound to procoagulant platelets concentrate in cap structures to promote clotting. Blood 2016;128:174555. https://doi.org/10.1182/blood-2016-02-696898.

7. Nechipurenko DY, Receveur N, Yakimenko AO, Shepelyuk TO, Yakusheva AA, Kerimov RR, et al. Clot Contraction Drives the Translocation of Procoagulant Platelets to Thrombus Surface. Arteriosclerosis, Thrombosis, and Vascular Biology 2019;39:37-47. https://doi.org/10.1161/ATVBAHA.118.311390.

8. Heemskerk JWM, Feijge MAH, Henneman L, Rosing $\mathrm{J}$, Hemker $\mathrm{HC}$. The $\mathrm{Ca}^{2+}$-mobilizing potency of a-thrombin and thrombin-receptor-activating peptide on human platelets Concentration and time effects of thrombin-induced $\mathrm{Ca}^{2+}$ signaling. European Journal of Biochemistry 1997;555:547-55.

9. Martyanov AA, Morozova DS, Sorokina MA, Filkova AA, Fedorova DV, Uzueva SS, et al. Heterogeneity of Integrin allbß3 Function in Pediatric Immune Thrombocytopenia Revealed by Continuous Flow Cytometry Analysis. IJMS 2020;21:3035. https://doi.org/10.3390/ijms21093035.

10. Sage SO, PUGH N, MASON MJ, HARPER AGS. Monitoring the intracellular store $\mathrm{Ca}^{2+}$ concentration in agonist-stimulated, intact human platelets by using Fluo-5N. Journal of Thrombosis and Haemostasis 2011;9:540-51.

11. Martyanov A, Panteleev M. Platelet functional responses and signalling: the molecular relationship. 
Part 2: receptors. SBPR 2021;1:13-30. https://doi. org/10.52455/sbpr.01.202103013.

12. Carafoli E, Krebs J. Why Calcium? How Calcium Became the Best Communicator. J Biol Chem 2016;291:20849 57. https://doi.org/10.1074/jbc.R116.735894.

13. Carafoli $E$. The calcium-signalling saga: tap water and protein crystals. Nat Rev Mol Cell Biol 2003;4:326-32. https://doi.org/10.1038/nrm1073.

14. Harper MT, Londoño JEC, Quick K, Londoño JC, Flockerzi V, Philipp SE, et al. Transient receptor potential channels function as a coincidence signal detector mediating phosphatidylserine exposure. SCIENCE SIGNALING 2013;6:1-11.

15. Sveshnikova AN, Balatskiy AV, Demianova AS, Shepelyuk TO, Shakhidzhanov SS, Balatskaya MN, et al. Systems biology insights into the meaning of the platelet's dual-receptor thrombin signaling. Journal of Thrombosis and Haemostasis 2016;14:2045-57. https://doi.org/10.1111/jth.13442.

16. Heemskerk JWM, Vis $P$, Feijge MAH, Hoylandll J, Mason WT. Roles of Phospholipase C and Ca2 ' -ATPase in Calcium Responses of Single , Fibrinogenbound Platelets *. The Journal of Biological Chemistry 1993;268:356-63.

17. Heemskerk JWM, Hoyland J, Masont WT, Sage S. Spiking in cytosolic calcium concentration in single fibrinogen-bound fura-2-loaded human platelets. BiochemJ 1992;283 ( Pt 2:379-83.

18. Obydennyy SI, Sveshnikova AN, Ataullakhanov $\mathrm{FI}$, Panteleev MA. Dynamics of calcium spiking, mitochondrial collapse and phosphatidylserine exposure in platelet subpopulations during activation. Journal of Thrombosis and Haemostasis 2016;14:1867-81. https://doi.org/10.1111/jth.13395.

19. Ha T, Tinnefeld P. Photophysics of Fluorescent Probes for Single-Molecule Biophysics and Super-Resolution Imaging. Annual Review of Physical Chemistry 2012;63:595-617. https://doi.org/10.1146/annurevphyschem-032210-103340.

20. Takahashi A, Camacho P, Lechleiter JD, Herman B. Measurement of Intracellular Calcium. Physiological Reviews 1999;79:1089-125. https://doi.org/10.1152/ physrev.1999.79.4.1089.

21. Balabin FA, Morozova DS, Mayorov AS, Martyanov AA, Panteleev MA, Sveshnikova AN. Clusterization of Inositol Trisphosphate Receptors Determines the Shape of the Calcium Oscillation Peak in Platelet Cytosol. Moscow Univ Phys 2018;73:526-33. https:// doi.org/10.3103/S0027134918050041.

22. Mazurov AV, Vinogradov DV, Kabaeva NV, Antonova GN, Romanov YA, Vlasik TN, et al. A Monoclonal Antibody, VM64, Reacts with a 130 kDa Glycoprotein Common to Platelets and Endothelial Cells: Heterogeneity in Antibody Binding to Human Aortic Endothelial Cells. Thromb Haemost 1991;66:494-9. https://doi.org/10.1055/s-0038-1646445.

23. Balabin FA. Quantitative Assessment of Heterogeneity of Single Platelet Calcium Responses to Activation. Res Pract Thromb Haemost 2021;5 (Suppl 2):PB1027.

24. Kokhan A, Zdanevich S, Prokofev I, Gorudko I, Shamova E. Patch-clamp technique for studying ion channels in activated platelets. Systems Biology and Physiology Reports 2021;1:3. https://doi.org/10.52455/ sbpr.01.202101012.

25. Mahaut-Smith MP. Calcium-activated potassium channels in human platelets. The Journal of Physiology 1995;484:15-24. https://doi.org/10.1113/jphysiol.1995. sp020644.

26. Thomas D, Tovey SC, Collins TJ, Bootman MD, Berridge MJ, Lipp P. A comparison of fluorescent Ca2+indicator properties and their use in measuring elementary and global $\mathrm{Ca}^{2+}$ signals. Cell Calcium 2000;28:213-23. https://doi.org/10.1054/ceca.2000.0152.

27. Thompson K, Dockery $\mathrm{P}$, Horobin R. Predicting and avoiding subcellular compartmentalization artifacts arising from acetoxymethyl ester calcium imaging probes. The case of fluo-3 AM and a general account of the phenomenon including a problem avoidance chart. Biotechnic \& Histochemistry 2012;87:468-83. https:// doi.org/10.3109/10520295.2012.703691.

28. Rink TJ, Pozzan T. Using quin2 in cell suspensions. Cell Calcium 1985;6:133-44. https://doi.org/10.1016/01434160(85)90040-5.

29. Nejadnik MR, Olsson ALJ, Sharma PK, van der Mei HC, Norde W, Busscher HJ. Adsorption of pluronic F-127 on surfaces with different hydrophobicities probed by quartz crystal microbalance with dissipation. Langmuir 2009;25:6245-9. https://doi.org/10.1021/la9001169.

30. Meyer F de, Smit B. Effect of cholesterol on the structure of a phospholipid bilayer. PNAS 2009;106:3654-8. https://doi.org/10.1073/pnas.0809959106.

31. Moscardó A, Vallés J, Latorre A, Santos MT. The association of thromboxane A2 receptor with lipid rafts is a determinant for platelet functional responses. FEBS Lett 2014;588:3154-9. https://doi.org/10.1016/j. febslet.2014.06.057.

32. Grgurevich S, Krishnan R, White MM, Jennings LK. Role of in vitro cholesterol depletion in mediating human platelet aggregation. J Thromb Haemost 2003;1:57686. https://doi.org/10.1046/j.1538-7836.2003.00087.x. 